\title{
19. COMMISSION DE LA VARIATION DES LATITUDES
}

\section{Compte-rendu de séance, 26 Août 1964}

Président: B. Guinot.

SECRÉTAIRE: R. O. Vicente.

Après avoir rappelé le décès de $\mathrm{L}$. Carnera et de $\mathrm{T}$. Hattori et fait adopter les Draft Reports, le Président consacre le début de la séance aux rapports sur les travaux du SIL et du SIMP.

T. Nicolini a accepté de discuter les observations de I94I à I 948 faites sous la direction de L. Carnera. Ce travail progresse régulièrement, malgré les difficultés qu'il y a à trouver les valeurs définitives des tours de vis; il est poursuivi suivant le plan de Carnera.

G. Cecchini n'a pas pu assister à la séance, mais une lettre récente assure également la Commission d'une bonne progression de la discussion des observations pour la période 1948 à I962.

Les travaux ont pu être poursuivis avec l'appui de la Commission Géodésique Italienne et aussi, signale $\mathrm{M}$. Tardi, grâce à l'aide financière de FAGS.

P. Tardi indique aussi que l'ICSU a contribué à la publication des observations de l'AGI faites par le SIL: les résultats seront extraits des fascicules de la Commission Géodésique Italienne. Cependant ces résultats ne concernent que les cinq stations internationales du SIL, il serait intéressant d'avoir l'ensemble des résultats de toutes les stations.

Le Président rappelle que la Commission ig avait déjà recommandé que les résultats des PZT soient publiés par l'Observatoire Naval de Washington, ceux des lunettes zénithales visuelles par l'Observatoire de Kiev et ceux des astrolabes par l'Observatoire de Paris. Les résultats des VZT ont déjà été publiés, mais ceux des PZT et des astrolabes ne sont pas complets.

N. Stoyko dit que le SIR peut publier des valeurs moyennes, mais non les valeurs individuelles.

Le Président propose une résolution invitant les observatoires à publier les résultats détaillés de leurs observations de latitude.

S. Yumi présente le rapport du SIMP, ainsi que le rapport annuel qu'il a publié suivant les statuts du SIMP (Annual Report of the IPMS for 1962, Mizusawa, 1964).

Dans ces rapports, la méthode 'latitude control' de W. Markowitz conduit à des résultats presque identiques à ceux de la méthode classique, aussi E. P. Fedorov propose-t-il de ne pas continuer avec les deux méthodes. Cependant, comme d'après S. Yumi, le 'latitude control' n'exige pas beaucoup de travail supplémentaire, le Président propose qu'on le poursuive jusqu'à la fin du présent programme d'observation, parce que la dissymétrie des distances zénithales s'accentue. P. Melchior propose qu'on essaie le 'latitude control' pour les observations faites sous la direction de Carnera.

Le Président rappelle qu'après le décès de T. Hattori, S. Yumi fut nommé Directeur intérimaire du Bureau Central du SIMP et propose une résolution pour recommander que $\mathrm{S}$. Yumi devienne Directeur de ce bureau. Cette résolution est adoptée à l'unanimité.

W. Markowitz présente ensuite ses travaux sur le mouvement séculaire du pôle et conclut à la réalité d'un déplacement annuel de $0^{\prime \prime}=003$ le long du méridien $55^{\circ} \mathrm{W}$. Cette conclusion est contestée par E. P. Fedorov, car la variation de la latitude d'une station contient un terme non 
polaire qui ne se réduit pas à un terme périodique et à une variation linéaire (due aux mouvements propres erronés) comme le montre la comparaison des résultats de deux stations de même longitude.

Le Président estime que ce problème ne peut pas être résolu sans un plus grand nombre de stations et rappelle la proposition de W. Markowitz d'associer les instruments par paires, à la même latitude (ou à quelques degrés de différence de latitude pour les astrolabes). Les astrolabes de Mizusawa et Alger ont déjà accordé leurs programmes.

N. Stoyko a trouvé, comme W. Markowitz, que la dérive du pôle est indépendante du choix des stations, du moins en ce qui concerne sa tendance générale.

S. Yumi distribue un article de S. Takagi sur l'usage simultané des mesures de temps et de latitude pour déterminer la polhodie.

\section{RÉSOLUTIONS ADOPTÉES}

\section{Texte français}

La Commission i9 de l'Union Astronomique Internationale

note avec satisfaction que le travail effectué par le Bureau Central du Service International du Mouvement Polaire, sous la direction intérimaire du Dr S. Yumi est d'une haute tenue scientifique,

prend acte des voeux de l'Association Internationale de Géodésie au sujet de la direction de ce bureau,

accepte avec gratitude l'offre du Conseil Scientifique du Japon de domicilier le Bureau Central du SIMP à Mizusawa,

propose de nommer le Dr S. Yumi, Directeur du Bureau Central du SIMP.

La Commission I 9

remercie la Commission Géodésique Italienne pour l'aide qu'elle apporte à la publication des résultats du Service International des Latitudes,

remercie le Prof. Nicolini pour avoir accepté de continuer le travail du regretté Prof. Carnera,

remercie le Prof. Cecchini pour la diligence avec laquelle il poursuit la discussion des observations effectuées sous sa direction.

La Commission ig

recommande que les observatoires participant à des observations suivies de la latitude publient le plus rapidement possible les résultats détaillés de leurs observations.

\section{Texte anglais}

Commission 19 of the International Astronomical Union

notes with satisfaction that the work carried out by the Central Bureau of the IPMS under the interim direction of Dr S. Yumi is of high scientific standard,

takes note of the resolution of the International Association of Geodesy concerning the direction of the Central Bureau,

accepts with gratitude the offer of the Scientific Council of Japan to locate the Central Bureau of the IPMS at Mizusawa and

nominates Dr S. Yumi to be Director of the Central Bureau of the IPMS.

Commission 19

thanks the Italian Geodetic Commission for the help it is giving in the publication of the results of the International Latitude Service, 
thanks Prof. Nicolini for having agreed to continue the work of the late Prof. Carnera, and

thanks Prof. Cecchini for the diligence with which he has pursued the discussion of the observations made under his direction.

Commission 19

recommends that observatories participating in latitude observation shall publish as quickly as possible the detailed results of their observations.

\section{Compte-rendu de la session mixte des Commissions rg et 31, 27 Août r964}

Président: Prof. A. A. Mikhailov.

SECRÉTAIRES: S. Débarbat et R. O. Vicente.

Le Président ouvre la séance qui sera consacrée à l'étude des projets de réorganisation des Commissions 19 et $3 \mathrm{I}$.

B. Guinot commente le projet de modification des champs d'activité qu'il a établi et distribué. Il y a de nombreuses raisons de fondre les Commissions 19 et $3^{\mathrm{I}}$, comme E. P. Fedorov l'avait proposé en $196 \mathrm{I}$ : on ne peut plus séparer la latitude du temps universel, ni dans leurs observations, ni dans leurs interprétations pour avoir le T.U.2 ou la polhodie, ni dans les problèmes théoriques de la rotation de la Terre. Il y a cependant des inconvénients, déjà mentionnés par W. Markowitz en I 961 , à grouper dans une même commission des problèmes aussi différents que ceux de l'observation astronomique, par exemple, et de la chronométrie. C'est pourquoi B. Guinot propose que la Commission I9, outre les problèmes dont elle s'occupe déjà, considère également ceux des observations du T.U., de leur synthèse et de leur interprétation. La nouvelle Commission i9 s'appellerait 'Commission de la Rotation de la Terre' (Rotation of the Earth). La nouvelle Commission 31, qui prendrait le nom de 'Commission du Temps' (Time) traiterait des problèmes de la conservation et de la diffusion du temps, ainsi que du Temps des Ephémérides.

A. A. Mikhailov propose une solution légèrement différente: les Commissions i9 et 3 I pourraient être fondues, mais on créerait deux comités techniques, du modèle suggéré par le Comité Exécutif de l'UAI, qui seraient constitués par le BIH et le SIMP.

La discussion est ouverte.

J. F. Denisse remarque que des services permanents comme le SIMP et le BIH ne correspondent pas exactement à la définition des comités techniques. D'ailleurs, D. H. Sadler, comme Secrétaire Général de l'Union, signale que le projet de création de comités techniques n'a pas été bien accueilli, en général, et qu'il préfèrerait qu'on n'en organise pas.

W. Markowitz signale que tous les membres du Comité d'Organisation de la Commission I 9 sont en accord avec le projet de B. Guinot.

P. Tardi soulève le problème des relations du BIH et du SIMP avec les nouvelles Commissions et avec l'UGGI. B. Guinot rappelle que la réorganisation des Commissions I9 et 3 I n'implique pas celle du SIMP et du BIH qui conservent leurs tâches respectives. Les activités du SIMP sont entièrement comprises dans celles de la nouvelle Commission ig. Celles du BIH sont réparties sur les deux Commissions, elles pourraient être discutées au sein de l'une ou de l'autre suivant leur nature. Cependant, pour éviter des répétitions, le rapport sur le fonctionnement du BIH sera fait à la Commission $3 \mathbf{r}$. Pour résoudre le problème des responsabilités de l'UGGI et de l'UAI, P. Melchior propose la création d'un conseil scientifique du BIH sur le modèle de celui du SIMP. W. Markowitz estime que c'est inutile, car le BIH a un rôle de coordination, mais non d'exécution comme le SIMP. Il suffit d'écrire à son directeur 
si l'on a à donner son avis sur son fonctionnement; toutefois, si on l'estime nécessaire, on pourrait utiliser le Comité d'Organisation de la Commission 3 I comme conseil du BIH.

Sur la proposition de H. M. Smith, le projet de B. Guinot est examiné point par point.

L. Essen remarque que parmi les sujets à traiter par la Commission $3 \mathrm{I}$, il y en a où l'UAI n'a pas pouvoir de décision: la définition de l'unité de temps physique, par exemple. W. Markowitz et B. Guinot répondent que même dans ces cas, la Commission $3^{I}$ doit examiner les décisions prises par d'autres organismes et en étudier les conséquences pour l'astronomie.

La responsabilité des Commissions vis-à-vis du Temps des Ephémérides est évoquée par D. H. Sadler. Il est reconnu qu'on ne demandera pas de modifications aux autres Commissions qui s'en occupent, mais que la Commission $3 \mathbf{r}$ aura à ce sujet un rôle de centralisation et de coordination des résultats.

Des modifications mineures de la liste des sujets à traiter par les nouvelles Commissions sont proposées par Randic et P. Tardi et acceptées par B. Guinot. En outre P. Tardi propose de conserver l'ancien nom français de 'Heure' pour la Commission $3 \mathbf{I}$ afin d'éviter toute ambiguité. P. Melchior inclut la nutation dans le domaine de la Commission 19.

D. H. Sadler n'est pas favorable à une limitation trop étroite des champs des Commissions et la liste de B. Guinot doit être considérée comme un guide.

Aucune objection n'étant plus élevée contre le projet de B. Guinot, ce projet est adopté. On trouvera en annexe la nouvelle répartition des tâches des Commissions 19 et $3 \mathbf{I}$.

\section{SUJETS À TRAITER PAR LES NOUVELLES COMMISSIONS I9 ET 3 I}

\section{Commission 19: Rotation de la Terre (Rotation of the Earth)}

(a) Déterminations astronomiques du T.U.o et de la latitude $a$ (I) instruments (construction, précision des résultats)

$a$ (2) méthodes (programmes d'observations, réductions)

(b) Synthèse et étude des mesures

$b$ (I) calcul de la polhodie d'après le temps et la latitude

$b$ (2) calcul de T.U.2 (différences T.U.2 - signaux horaires) d'après les mesures de T.U.O et de latitude

$b$ (3) analyses et interprétations théoriques de la polhodie et des irrégularités de la rotation terrestre (T.U.2 - temps atomique)

$b(4)$ variations séculaires des latitudes et des longitudes

$b$ (5) mouvements locaux réels ou apparents de la verticale (réfractions zénithales, réfractions de salle, marées)

$b(6)$ étude de la nutation

Commission 3 I : Heure (Time)

(c) Définition du temps

$c$ (I) unités de temps

$c$ (2) définition des échelles de temps astronomiques (T.U.O, T.U.I, T.U.2, T.E.)

(d) Observations astronomiques du Temps des Ephémérides

$d(\mathrm{I})$ instruments

$d(2)$ méthodes (programmes, réductions)

(e) Chronométrie

$e$ (I) horloges, étalons de fréquences

$e(2)$ appareils auxiliaires: chronographes, etc. 
(f) Diffusion du temps:

diffusion des fréquences avec éventuels décalages signaux horaires en T.U., forme, sauts

coordination des émissions de fréquences et des signaux horaires

(g) Comparaisons entre le T.E. et le temps atomique

(valeur de la fréquence des étalons atomiques, rapportée à la seconde des éphémérides).

\section{Réunion du Conseil Scientifique du Service International du Mouvement Polaire}

Ce conseil, au complet, s'est réuni le 26 Août I 964 et a examiné les problèmes pratiques posés par l'usage des stations libres et des mesures de temps pour le calcul de la polhodie. 